DOI: $10.24850 /$ j-tyca-2021-01-09

Artículos

\title{
Comparación de los modelos WEAP y SWAT en una cuenca de Oaxaca
}

\section{Comparison between WEAP and SWAT models in a basin at Oaxaca, Mexico}

María Magdalena Nevárez-Favela ${ }^{1}$

Demetrio Salvador Fernández-Reynoso²

Ignacio Sánchez-Cohen ${ }^{3}$

Madaí Sánchez-Galindo 4

Antonia Macedo-Cruz 5

Carlos Palacios-Espinosa 6

${ }^{1}$ Colegio de Postgraduados, Campus Montecillo, Montecillo, Estado de México, México, nevarez.magdalena@colpos.mx

${ }^{2}$ Colegio de Postgraduados, Campus Montecillo, Montecillo, Estado de México, México, demetrio@colpos.mx

3Instituto Nacional de Investigaciones Forestales, Agrícolas y PecuariasCentro Nacional de Investigación Disciplinaria en Relación Agua, Suelo, 
Planta, Atmósfera, Gómez Palacio, Durango, México, sanchez.ignacio@inifap.gob.mx

${ }^{4}$ Colegio de Postgraduados, Campus Montecillo, Montecillo, Estado de México, México, sanchez.madai@colpos.mx

${ }^{5}$ Colegio de Postgraduados, Campus Montecillo, Montecillo, Estado de México, México, macedoan@colpos.mx

${ }^{6}$ Consultora KANKI, Texcoco, Estado de México, México, c.palacios.e@gmail.com

Autora para correspondencia: Madaí Sánchez-Galindo, sanchez.madai@colpos.mx

\section{Resumen}

La cuenca del río Sordo, localizada al oeste de Oaxaca, México, presenta problemas de erosión hídrica y pérdida de biodiversidad. El presente trabajo tiene como objetivo comparar los escurrimientos aforados en la estación Ixtayutla (20021), con valores simulados de los modelos WEAP (Water Evaluation And Planning System) y SWAT (Soil and Water Assessment Tool). Se procuró que WEAP, a través del método de la humedad del suelo, utilizara los mismos datos climáticos, de vegetación y suelos que SWAT, reportados para esta misma cuenca por SánchezGalindo, Fernández-Reynoso, Martínez-Ménez, Rubio-Granados y RíosBerber (2017). La comparación se basó en la eficiencia estadística de 
ambos modelos para simular los escurrimientos mensuales y anuales ocurridos durante el periodo 1975-1985. Se calcularon tres índices de eficiencia: el coeficiente de determinación $\left(r^{2}\right)$, Nash-Sutcliffe (NSE) y el sesgo porcentual (PBIAS). Con respecto a los escurrimientos mensuales aforados, WEAP presentó un NSE $=0.73$ (bueno); un PBIAS $=-16.05$ (satisfactorio), y una $r^{2}=0.84$. SWAT, para ese mismo periodo, mostró un NSE $=0.82$ (muy bueno); un PBIAS $=-15.92$ (satisfactorio), y una $r^{2}$ $=0.85$. Para los escurrimientos anuales, SWAT y WEAP obtuvieron un NSE de 0.73 y 0.3 , un $r^{2}$ de 0.76 y 0.63 y un PBIAS de -4.65 y -16.23 , respectivamente. Los dos modelos resultaron satisfactorios para simular escurrimientos mensuales, por lo que la elección de uno u otro modelo dependerá de la problemática de la cuenca, los datos con que se cuente y los objetivos por cumplir.

Palabras clave: SWAT, método de la humedad del suelo, Mixteca oaxaqueña, Nash-Sutcliffe, cuencas hidrográficas.

\section{Abstract}

The Sordo Basin is located in the western portion of the state of Oaxaca, Mexico. This presents problems of water erosion and loss of biodiversity. The present paper aims to compare the measured runoffs at the Ixtayutla station (20021) with simulated runoff by the WEAP model (Water Evaluation and Planning) and the results of the SWAT (Soil and Water Assessment Tool) model reported for the same basin by Sánchez-Galindo, 
Fernández-Reynoso, Martínez-Ménez, Rubio-Granados and Ríos-Berber (2017). WEAP-Soil Moisture Method used the same weather data, land use and soil type than SWAT. The comparison was based on the statistical efficiency of both models to simulate the monthly and annual runoff during the period 1975-1985. Three efficiency indices were calculated: the coefficient of determination $\left(r^{2}\right)$, Nash-Sutcliffe efficiency (NSE) and the percent bias (PBIAS). Regarding the monthly runoffs, WEAP presented a NSE $=0.73$ (good); a PBIAS $=-16.05$ (satisfactory), and $r^{2}=0.84$. $S W A T$, for that same period, reaching a NSE $=0.82$ (very good); a PBIAS $=-15.92$ (satisfactory), and a $r^{2}=0.85$. For annual runoffs, SWAT y WEAP getting a NSE $=0.73$ and $0.3, a r^{2}=0.76$ and 0.63 and a PBIAS $=-4.65$ and -16.23 , respectively. Both models are satisfactory to simulate monthly runoffs and the choice between one or other will depend on the problems to study in the basin, the available data and the hydrological goals.

Keywords: SWAT, Soil Moisture Method, Mixteca oaxaqueña, NashSutcliffe, watersheds.

Recibido: 08/02/2020

Aceptado: $15 / 06 / 2020$ 
Tecnología y

Ciencias Agua
2021, Instituto Mexicano de Tecnología del Agua

Open Access bajo la licencia CC BY-NC-SA 4.0

(https://creativecommons.org/licenses/by-nc-sa/4.0/)

\section{Introducción}

En el mundo, el deterioro de los recursos naturales es cada vez más grave. Las causas de este problema pueden ser tanto naturales como antrópicas. Sin embargo, la sociedad debe actuar para conocer y evaluar la interacción entre el comportamiento humano y el estado de los recursos, sobre todo cuando el crecimiento económico y poblacional trae consigo la necesidad de más recursos naturales.

En México, el estado de Oaxaca es rico en diversidad de recursos naturales, pero con alta presión de uso. Específicamente, la región Mixteca presenta una fuerte degradación de sus suelos y de su cubierta vegetal. La cuenca del río Sordo, tributaria del río Verde, que desemboca al océano Pacífico, abarca una superficie de $7751.42 \mathrm{~km}^{2}$, que cubre el $54 \%$ de la Mixteca oaxaqueña (Sánchez-Galindo et al., 2017).

La cuenca del río Sordo está cubierta principalmente por material vulcano-sedimentario (70 \%); presenta un relieve escarpado (pendiente media, $36.3 \%$ ), y es azotada por lluvias de alta intensidad (46 \pm 13.3 $\mathrm{mm} \mathrm{hr}^{-1}$, media anual) provenientes de huracanes tropicales. La presencia de materiales sedimentarios no consolidados, lo escarpado del relieve, la 
presencia de Iluvias ciclónicas y la agricultura en laderas favorecen los procesos erosivos y pérdida de la capacidad del suelo para retener humedad. Sin embargo, la intervención del hombre ha acelerado la degradación del suelo y disminuido la densidad de la cubierta vegetal por efecto del sobrepastoreo, el mal aprovechamiento forestal y la agricultura en ladera (Sánchez-Galindo et al., 2017).

La energía de la lluvia derivada de su intensidad, y la energía potencial que aporta la topografía, favorecen el desprendimiento de partículas de suelo por el impacto de las gotas y el atrincheramiento de cauces por la concentración de escurrimientos de alta velocidad sobre depósitos sedimentarios. Los procesos de degradación por efecto del escurrimiento superficial y la degradación de la cubierta vegetal hacen necesario comprender a detalle los procesos relacionados con el recurso hídrico que ocurren en la cuenca del río Sordo. En este sentido, los modelos de simulación son una herramienta útil para identificar relaciones de causa y efecto. Para ello, en la presente investigación se utilizó la misma información ambiental y parámetros calibrados en SWAT para correr el modelo WEAP. Esto, con el propósito de complementar el análisis hidrológico de la cuenca con las capacidades de WEAP y su vez conocer la respuesta del modelo al simular la misma serie histórica de aforos empleados durante la calibración de SWAT.

Los modelos hidrológicos son las herramientas más utilizadas para el análisis de los procesos naturales que ocurren en una cuenca (Singh \& Woolhiser, 2002). Estos modelos son representaciones de los 
componentes biofísicos de una cuenca, que simulan con cierto grado de confianza las diversas salidas del ciclo hidrológico (Salgado \& Güitrón, 2012).

Entre los modelos usados para el análisis hidrológico de cuencas sobresalen SWAT y WEAP. El modelo SWAT es un modelo de tiempo continuo, semidistribuido, basado en procesos, desarrollado para evaluar estrategias de gestión sobre recursos hídricos y contaminación de fuentes no puntuales en grandes cuencas. Su directriz es el balance hídrico, el cual afecta el crecimiento de las plantas y el movimiento de sedimentos, nutrientes, pesticidas y patógenos (Cuceloglu, Abbaspour, \& Ozturk, 2017). Por otra parte, WEAP es un modelo híbrido conceptual-base física y de un número reducido de parámetros, que simula los componentes naturales e intervenidos de los sistemas de recursos hídricos, que ha sido aplicado en cuencas de diversos tamaños y es adecuado para la evaluación de escenarios (Hernández-Vargas, 2017).

En 2017, Sánchez-Galindo et al. elaboraron un estudio en la cuenca del río Sordo, Oaxaca, con el modelo hidrológico SWAT, en el cual evaluaron la eficiencia de dicha herramienta para simular biomasa, escurrimientos y sedimentos para el periodo 1975-1985. En este trabajo se optó por comparar las bondades del modelo WEAP para simular, en ese mismo periodo y sin calibrar parámetros, los escurrimientos aforados a través de la información empleada y generada con SWAT. El propósito de utilizar la información calibrada con SWAT es observar el desempeño de un modelo como WEAP con una conceptualización hidrológica diferente 
a SWAT en el cálculo del escurrimiento superficial, infiltración, percolación, y flujo superficial y base; complementar el análisis hidrológico de la cuenca con procesos no incluidos en SWAT, como la evapotranspiración a través de coeficientes de cultivo y el movimiento de agua con valores de conductividad hidráulica; y comparar la respuesta de $W E A P$, con menores requerimientos de información y en igualdad de datos de entrada, con los escurrimientos aforados y usados en la calibración con SWAT.

Es factible obtener resultados satisfactorios en WEAP utilizando parámetros calibrados en SWAT, como se ha realizado en otras investigaciones. Los modelos SWAT y WEAP se han utilizado conjuntamente en cuencas de Etiopía y Lesoto. El primero, para conocer el sistema y su comportamiento hidrológico; mientras que el segundo, utilizando resultados de SWAT para cuantificar la distribución de los recursos hídricos de la cuenca bajo diversos criterios (Adgolign, SrinivasaRao, \& Abbulu, 2016; Hussen, Mekonnen, \& Pingale, 2018; Maliehe \& Mulungu, 2017).

\section{Materiales y métodos}


Tecnología y

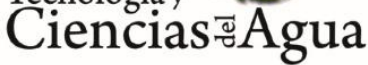

2021, Instituto Mexicano de Tecnología del Agua

Open Access bajo la licencia CC BY-NC-SA 4.0

(https://creativecommons.org/licenses/by-nc-sa/4.0/)

\section{Área de estudio}

La cuenca del río Sordo se localiza en el estado de Oaxaca, entre los paralelos $17^{\circ} 37^{\prime} 19.93^{\prime \prime}$ y $16^{\circ} 29^{\prime} 43.11^{\prime \prime}$ de latitud norte, y los meridianos $98^{\circ} 05^{\prime} 54.34^{\prime \prime}$ y $96^{\circ} 53^{\prime} 17.86^{\prime \prime}$ de longitud oeste. Cuenta con una altitud que va desde los 274 msnm hasta los 3349 msnm, y comprende una superficie de $7751.42 \mathrm{~km}^{2}$, en la que confluyen varios ríos; los más importantes por su dimensión son Peñoles, Labor, Cuchara, Zapote, Yolotepec y Sordo. El río Sordo-Yolotepec desemboca en la estación hidrométrica Ixtlayutla (20021) (Figura 1). 
2021, Instituto Mexicano de Tecnología del Agua

Tecnología y tiv

Ciencias $₫$ Agua

Open Access bajo la licencia CC BY-NC-SA 4.0

(https://creativecommons.org/licenses/by-nc-sa/4.0/)
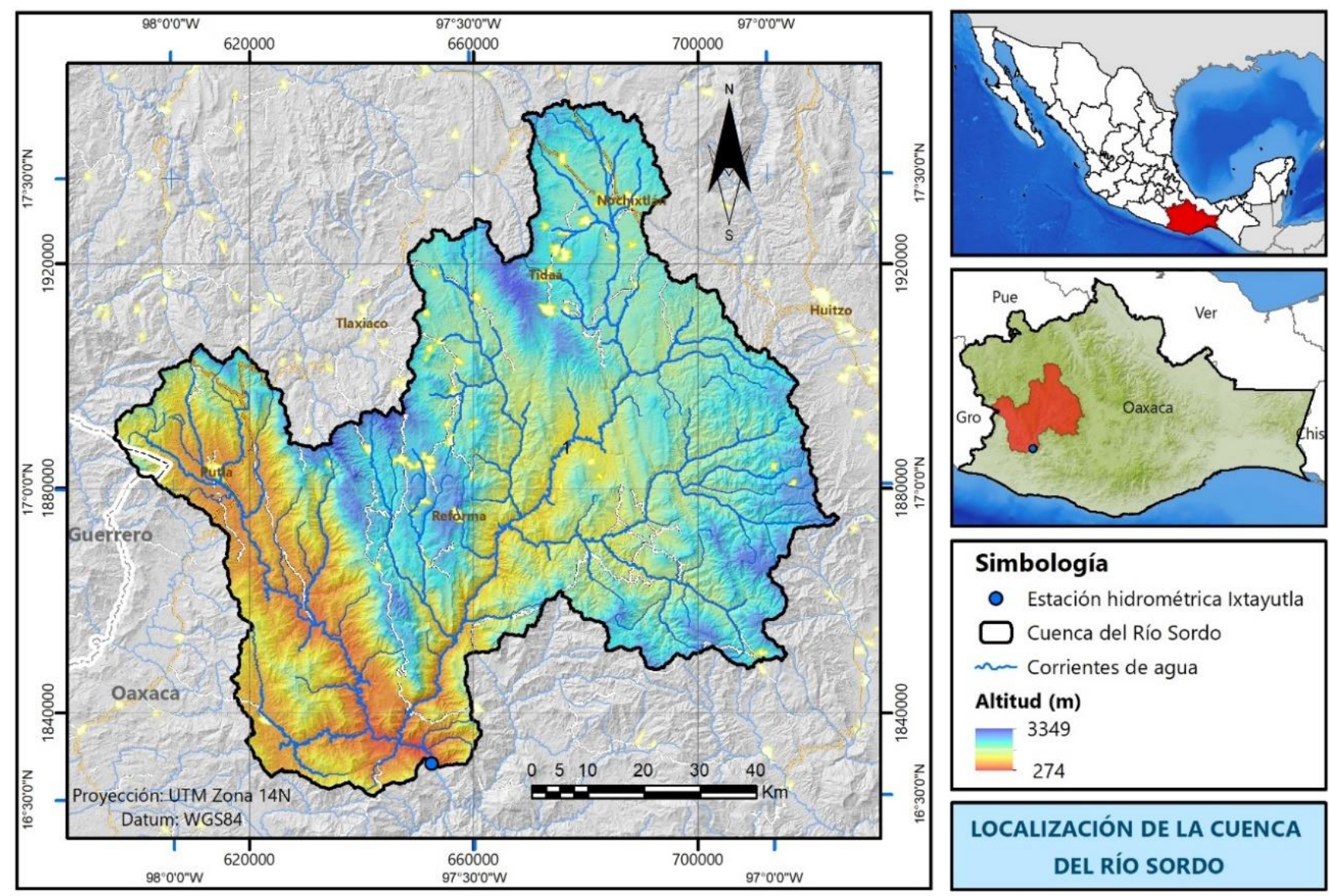

Simbología

- Estación hidrométrica Ixtayutla

$\square$ Cuenca del Río Sordo

m Corrientes de agua

Altitud ( $m$ )

3349

274

LOCALIZACIÓN DE LA CUENCA DEL RÍO SORDO

Figura 1. Localización de la cuenca del río Sordo, Oaxaca, México.

Esta cuenca abarca cuatro regiones culturales: Mixteca (54.4\%), Sierra Sur (30.6\%), Valles Centrales (11.7\%) y Costa (3.3\%). Se sitúa sobre dos acuíferos principales: Nochixtlán $\left(1321.84 \mathrm{~km}^{2}\right)$ y Jamiltepec (6 $269.18 \mathrm{~km}^{2}$ ). Los climas son templado húmedo y subhúmedo (48.6 $\%)$, semicálido subhúmedo $(34.0 \%)$, cálido subhúmedo (16.0\%) y semiseco templado (1.4\%). Registra temperaturas medias anuales que 
oscilan entre 10 y $28{ }^{\circ} \mathrm{C}$. Muestra precipitaciones de $400 \mathrm{~mm}$ en el norte, que se incrementan hasta $1600 \mathrm{~mm}$ en la parte sur. Presenta nueve tipos de suelos: cambisol (22.0 \%), rendzina (20.3\%), acrisol (15.4\%), vertisol (10.4\%), litosol (7.7 \%), fluvisol (7.5\%), luvisol $(6.9 \%)$, phaeozem (6.7\%) y regosol (3.1\%). Además, tiene 13 tipos de uso de suelo y vegetación: bosque de pino-encino (23.1\%), bosque de pino (20.6\%), pastizal (18.5\%), bosque de encino (15.6\%), agricultura de temporal $(10.7 \%)$, selva baja caducifolia $(6.4 \%)$, chaparral $(2.1 \%)$, bosque de encino-pino (1.3\%), bosque mesófilo de montaña $(0.7 \%)$, asentamientos humanos $(0.5 \%)$, bosque de táscate $(0.2 \%)$, cuerpos de agua $(0.1 \%)$ y agricultura de riego $(0.1 \%)$.

\section{Método de la humedad del suelo (WEAP)}

De los cinco métodos que WEAP posee para realizar el balance hídrico, se seleccionó el método de la humedad del suelo (Soil Moisture Method). Este método representa a la cuenca a través de dos capas de suelo; caracteriza la cubierta vegetal y el tipo de suelo; y mediante funciones empíricas estima la evapotranspiración, escurrimiento superficial, 
escurrimiento subsuperficial y percolación profunda (Sieber \& Purkey, 2015; Yates, Sieber, Purkey, \& Huber-Lee, 2005).

En WEAP, la cuenca puede ser dividida en subcuencas, las cuales, a su vez, pueden ser divididas en $N$ áreas con diferentes cubiertas vegetales $j$. El balance de agua en la zona radicular y en la zona profunda se calculan por tipo de cobertura con la Ecuación (1) y Ecuación (2), respectivamente (Yates et al., 2005):

$$
\begin{aligned}
& S w_{j} \frac{d z_{1, j}}{d t}=P_{e}(t)-P E T(t) K c_{, j}(t)\left(\frac{5 z_{1, j}-2 z_{1, j}^{2}}{3}\right)-P_{e}(t) \frac{\frac{L A I_{j}}{z_{1, j}^{2}}}{3}-f_{j} k_{j} z_{1, j}^{2}-\left(1-f_{j}\right) k_{j} z_{1, j}^{2} \\
& D w \frac{d z_{2, j}}{d t}=\left(1-f_{j}\right) k_{j} z_{1, j}^{2}-k_{2} z_{2, j}^{2}
\end{aligned}
$$

Donde $S w_{j}$ es la capacidad de almacenamiento de agua en la zona radicular del suelo $(\mathrm{mm}) ; z_{1, j}$, es el almacenamiento relativo de agua en la zona radicular del suelo, dado como una fracción del almacenamiento total efectivo $(1,0) ; P_{e}(t)$, precipitación efectiva en el tiempo $t(\mathrm{~mm})$; $\operatorname{PET}(t)$, evapotranspiración potencial del cultivo de referencia de PenmanMontieth ( $\left.\mathrm{mm} \mathrm{tiempo-1}^{-1}\right) ; K c_{, j}(t)$, coeficiente de cultivo en el tiempo $t$ (adimensional); $L A I_{j}$, índice de área foliar $\left(\mathrm{m}^{2} \mathrm{~m}^{-2}\right)$ (el escurrimiento decrece conforme aumenta este valor); $f_{j}$, parámetro de ajuste cuasi- 
físico relacionado con el tipo de suelo, uso de suelo y vegetación, y topografía que direcciona el agua ya sea horizontalmente $\left(f_{j}\right)$ o verticalmente $\left(1-f_{j}\right)(1.0=100 \%$ horizontal, $0=100 \%$ vertical $) ; k_{j}$, un estimado de la conductividad del almacenamiento en la zona radicular ( $\mathrm{mm}$ tiempo-1); $D w$, capacidad de almacenamiento de agua en la capa profunda del suelo $(\mathrm{mm}) ; z_{2, j}$, almacenamiento relativo de agua en la capa profunda del suelo dado como una fracción del almacenamiento total efectivo $(1,0)$ (Figura 2).

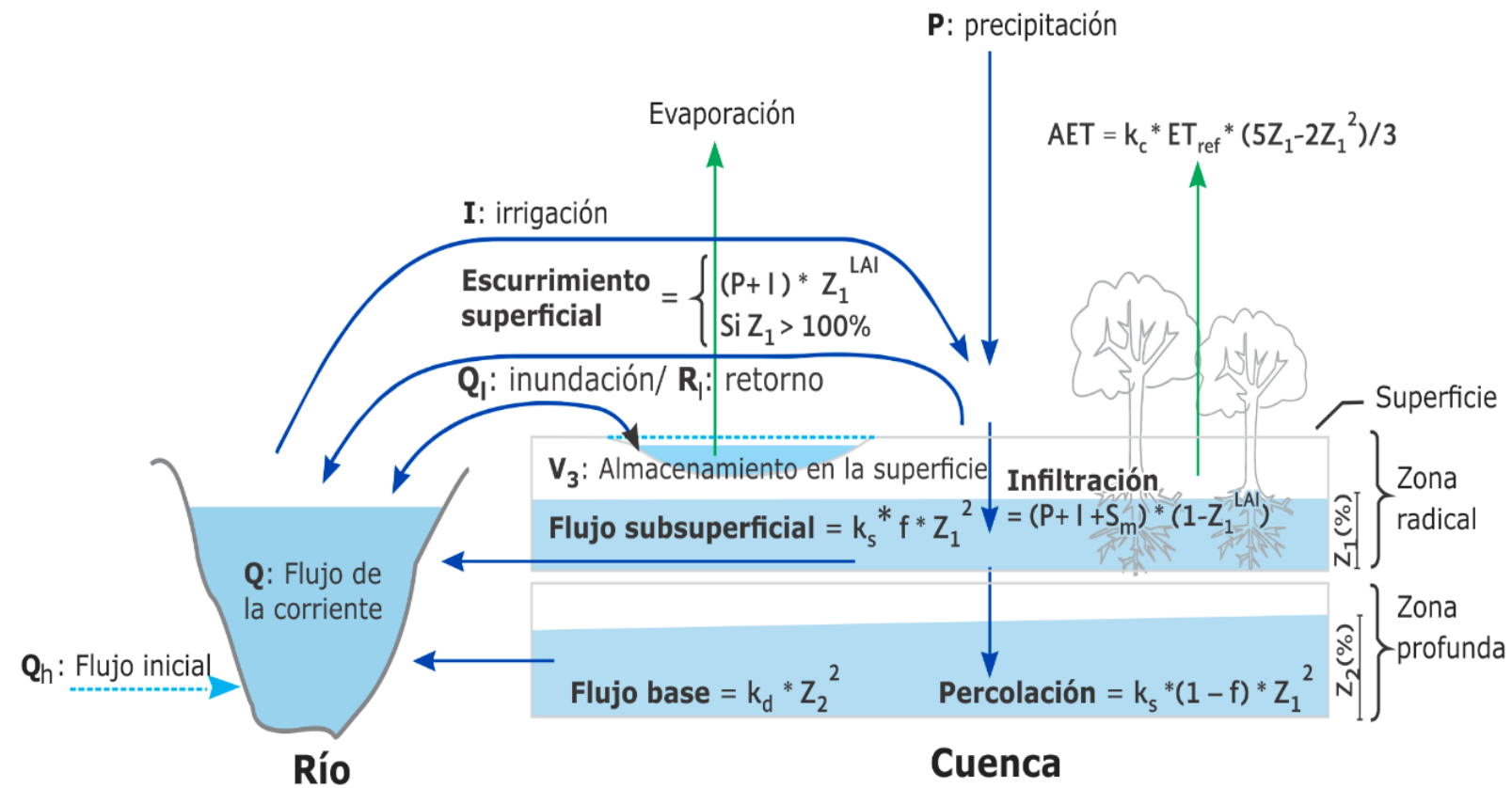

Figura 2. Esquema del método de la humedad del suelo (Angarita et

$$
\text { al., 2018). }
$$




\section{Alimentación del modelo}

La metodología empleada para la alimentación de WEAP se muestra en la Figura 3. Los datos generados en SWAT para las unidades de respuesta hidrológica (HRU por sus siglas en inglés) de la cuenca del río Sordo calibrados por Sánchez-Galindo et al. (2017) se incorporaron, previa ponderación entre superficies y conversión de unidades, a las áreas (branches) con que opera WEAP. 


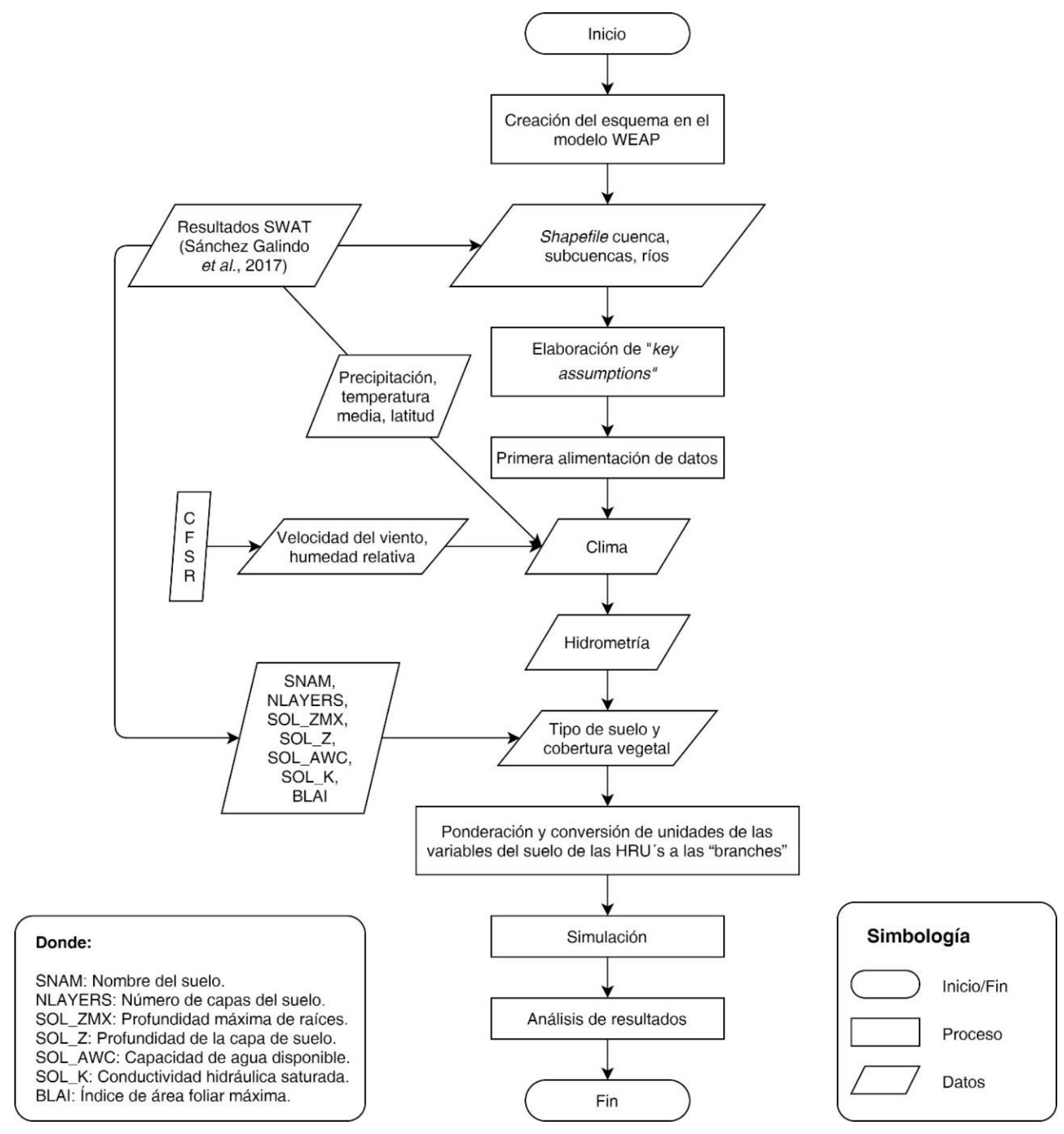

Figura 3. Metodología utilizada en el modelo WEAP para la cuenca del río Sordo. 
Para operar WEAP, se integró el esquema general de la cuenca, con base en la delimitación empleada en SWAT, a través de 175 subcuencas con sus cauces principales y la estación hidrométrica 20021 "Ixtayutla". Posteriormente, en Data $\rightarrow$ Demand Sites and Catchments se agregaron manualmente las ramificaciones, 1 729, es decir, las unidades de respuesta hidrológica creadas por SWAT, utilizándose para ello la tabla generada en la capa vectorial "FullHRU".

Después del esquema, se crearon los supuestos clave (key assumptions) para las variables: precipitación, temperatura media, latitud, velocidad del viento, humedad relativa, coeficiente de cultivo, capacidad de almacenamiento de agua en la zona radical, índice de área foliar (factor de resistencia al escurrimiento), conductividad hidráulica en la zona radical y dirección preferencial de flujo. Los supuestos clave se utilizan cuando se trabaja con muchas subcuencas que requieren ingresar la misma información. Esta herramienta, junto con las opciones Export expressions to Excel e Import expressions from Excel, localizadas en la ventana Edit, agilizaron el ingreso de datos.

La recopilación y acomodo de los datos climáticos, hidrométricos y de cobertura vegetal/tipo de suelo se describe a continuación.

De la hoja wgn del libro Access, nombrado como el proyecto creado en SWAT, se identificaron las estaciones meteorológicas empleadas en SWAT para la cuenca del río Sordo (Tabla 1). 
Tecnología y

Ciencias $₫$ Agua
2021, Instituto Mexicano de Tecnología del Agua

Open Access bajo la licencia CC BY-NC-SA 4.0

(https://creativecommons.org/licenses/by-nc-sa/4.0/)

Tabla 1. Estaciones climatológicas utilizadas en la modelación hidrológica de la cuenca del río Sordo.

\begin{tabular}{|c|c|c|c|c|}
\hline Clave & Nombre & $\begin{array}{l}\text { Latitud } \mathbf{N} \\
\left({ }^{\circ}\right)\end{array}$ & $\begin{array}{c}\text { Longitud } 0 \\
\left({ }^{\circ}\right)\end{array}$ & $\begin{array}{l}\text { Altitud } \\
\text { (m) }\end{array}$ \\
\hline 20026 & $\begin{array}{l}\text { Chalcatongo de } \\
\text { Hidalgo }\end{array}$ & 17.03300 & -97.58300 & 2250 \\
\hline 20038 & Santiago Ixtayutla & 16.56700 & -97.66700 & 510 \\
\hline 20044 & Jalapa del Valle & 17.06700 & -96.88300 & 1650 \\
\hline 20076 & $\begin{array}{l}\text { Asunción Nochixtlán } \\
\text { (SMN) }\end{array}$ & 17.46667 & -97.21667 & 2080 \\
\hline 20094 & $\begin{array}{l}\text { Putla de Guerrero } \\
\text { (CFE) }\end{array}$ & 17.11667 & -97.87305 & 1316 \\
\hline 20102 & $\begin{array}{l}\text { San Agustín } \\
\text { Tlacotepec }\end{array}$ & 17.20000 & -97.51778 & 2018 \\
\hline 20105 & $\begin{array}{l}\text { San Esteban } \\
\text { Atatlahuaca }\end{array}$ & 17.06500 & -97.67917 & 2455 \\
\hline 20126 & Sta. Cruz Zenzotepec & 16.53300 & -97.48300 & 970 \\
\hline 20130 & Sta. María Yucuhiti & 17.01667 & -97.79972 & 1876 \\
\hline 20153 & $\begin{array}{l}\text { Sto. Domingo } \\
\text { Teojomulco }\end{array}$ & 16.60000 & -97.21700 & 1300 \\
\hline
\end{tabular}


Tecnología y

Ciencias $₫$ Agua
2021, Instituto Mexicano de Tecnología del Agua

Open Access bajo la licencia CC BY-NC-SA 4.0

(https://creativecommons.org/licenses/by-nc-sa/4.0/)

\begin{tabular}{|l|l|c|c|c|}
\hline Clave & \multicolumn{1}{|c|}{ Nombre } & $\begin{array}{c}\text { Latitud N } \\
\mathbf{(}^{\circ}\end{array}$ & \multicolumn{1}{|c|}{$\begin{array}{c}\text { Longitud O } \\
\mathbf{(}^{\circ} \mathbf{)}\end{array}$} & $\begin{array}{c}\text { Altitud } \\
\mathbf{( m )}\end{array}$ \\
\hline 20159 & Pedro y Pablo Teposcol & 17.50131 & -97.48254 & 2183 \\
\hline 20167 & $\begin{array}{l}\text { Sta. Ma. Asunción } \\
\text { Tlax. (DGE) }\end{array}$ & 17.26700 & -97.68300 & 2065 \\
\hline 20178 & $\begin{array}{l}\text { Villa Chalcatongo, } \\
\text { (CFE) }\end{array}$ & 17.03306 & -97.58305 & 2428 \\
\hline 20186 & $\begin{array}{l}\text { Santiago Yosondua, } \\
\text { Stgo. Y. }\end{array}$ & 16.89972 & -97.59972 & 2222 \\
\hline 20187 & $\begin{array}{l}\text { Yutacua, } \\
\text { Ixtayutla }\end{array}$ & 16.60361 & -97.62500 & 437 \\
\hline Zacatepec, Zacatepec & 16.75000 & -97.78300 & 900 \\
\hline
\end{tabular}

Los datos diarios de precipitación y temperatura ingresados en el modelo SWAT se pasaron a valores mensuales. En específico, para WEAP se tienen los datos mensuales de velocidad del viento (VV) y humedad relativa (HR) entre 1979 y 1985, que se obtuvieron para la malla que utiliza a nivel global el Climate Forecast System Reanalysis (NCEP, 2019). Los datos de HR y VV, para la malla que corresponde a la cuenca, se interpolaron a nivel mensual (con la extensión Spline de ArcMap) y se extrajeron los datos específicos para coordenadas geográficas de las estaciones mostradas en la Tabla 1. 
Los datos de escurrimiento usados en la calibración y validación de SWAT, obtenidos del Banco Nacional de Datos de Aguas Superficiales (Conagua-IMTA, 2019) para la estación Ixtayutla (Sánchez-Galindo et al., 2017), se ingresaron mensualmente a WEAP mediante la ruta Supply and Resources $\rightarrow$ River $\rightarrow$ "Sordo" $\rightarrow$ Streamlow Gauges $\rightarrow$ "Ixtayutla" $\rightarrow$ ReadFromFile Wizard.

Enseguida se describen las variables de vegetación/suelo que componen el modelo WEAP. La metodología del coeficiente del cultivo $\left(K_{c}\right)$ se usó para calcular la evapotranspiración de cultivo bajo condiciones estándar $\left(E T_{C}\right)$ (ver Ecuación (3)). Las condiciones estándar son aquellas que se desarrollan en campos extensos, bajo condiciones agronómicas excelentes y sin limitaciones de humedad del suelo. La evapotranspiración de un cultivo $\left(E T_{C}\right)$ discrepa de la de referencia $\left(E T_{o}\right)$, obtenida por lo general para un pasto, en función de las características de cobertura del suelo, propiedades de la vegetación y resistencia aerodinámica; efectos que están incorporados en el coeficiente del cultivo $K_{c}$ (Allen, Pereira, Raes, \& Smith, 2006) (Tabla 2):

$E T_{c}=K_{c} E T_{o}$

Tabla 2. Valores de $K c$ utilizados en la cuenca del río Sordo. Fuente: Hernández-Vargas (2017). 
Tecnología y

Ciencias $\stackrel{\gtrsim}{\triangleleft}$ gua
2021, Instituto Mexicano de Tecnología del Agua

Open Access bajo la licencia CC BY-NC-SA 4.0

(https://creativecommons.org/licenses/by-nc-sa/4.0/)

\begin{tabular}{|l|l|c|}
\hline Clave & \multicolumn{1}{|c|}{ Descripción } & Coeficiente de cultivo (KC) \\
\hline BENC & Bosque de encino & 0.9 \\
\hline ENPI & Bosque de encino-pino & 0.8 \\
\hline FRSD & Selva baja caducifolia & 1.0 \\
\hline FRSE & Bosque mesófilo de montaña & 0.6 \\
\hline MATO & Chaparral & 1.0 \\
\hline PASI & Pastizal & 1.0 \\
\hline PIEN & Bosque de pino-encino & 1.0 \\
\hline PINO & Bosque de pino & 1.1 \\
\hline RIEG & Agricultura de riego & 0.8 \\
\hline RNGB & Bosque de táscate & 0.9 \\
\hline TEMP & Agricultura de temporal & 1.0 \\
\hline URMD & Residencial mediana densidad & 0.7 \\
\hline WATR & Cuerpos de agua & \\
\hline
\end{tabular}

Como ya se indicó, una de las características más distintivas del método de la humedad del suelo es que representa a la cuenca a través de dos capas de suelo. Por consiguiente, la profundidad de la primera capa por tipo de suelo (Ecuación (4)) se obtuvo ponderando las profundidades radicales de referencia de las coberturas vegetales contenidas en la Tabla 3 (valores adquiridos del trabajo de Sánchez- 
Galindo et al. (2017), con base en datos predeterminados en SWAT). La profundidad de la capa radical por tipo de suelo, es decir, las ponderaciones resultantes, se redondearon a múltiplos de 50 (Tabla 4). Por otra parte, el grosor de la zona profunda de cada tipo de suelo se obtuvo con la diferencia entre los valores de profundidad total (datos obtenidos por Sánchez-Galindo et al. (2017) de las capas de perfiles de suelo, serie II, del INEGI) y de la primera capa (Tabla 4):

$P r_{p}=\sum_{i=1}^{n} \frac{A_{i} U S V_{i}}{A T_{S}}$

Donde $P r_{p}$ es la profundidad radicular ponderada $(\mathrm{mm}) ; A_{i}$, el área del uso de suelo y vegetación (ha); $U S V_{i}$, la profundidad de la vegetación $(\mathrm{mm}) ;$ y $A T_{s}$ es el área total del tipo de suelo de interés (ha).

Tabla 3. Profundidades radicales para cada uso de suelo y vegetación de la cuenca del río Sordo.

\begin{tabular}{|l|l|c|}
\hline Clave & \multicolumn{1}{|c|}{ Descripción } & Profundidad (mm) \\
\hline BENC & Bosque de encino & 600 \\
\hline ENPI & Bosque de encino-pino & 600 \\
\hline FRSD & Selva baja caducifolia & 500 \\
\hline FRSE & Bosque mesófilo de montaña & 1000 \\
\hline
\end{tabular}


Tecnología y

Ciencias $₫$ Agua
2021, Instituto Mexicano de Tecnología del Agua

Open Access bajo la licencia CC BY-NC-SA 4.0

(https://creativecommons.org/licenses/by-nc-sa/4.0/)

\begin{tabular}{|l|l|c|}
\hline Clave & \multicolumn{1}{|c|}{ Descripción } & Profundidad (mm) \\
\hline MATO & Chaparral & 400 \\
\hline PASI & Pastizal & 600 \\
\hline PIEN & Bosque de pino-encino & 800 \\
\hline PINO & Bosque de pino & 600 \\
\hline RIEG & Agricultura de riego & 500 \\
\hline RNGB & Bosque de táscate & 350 \\
\hline TEMP & Agricultura de temporal & 650 \\
\hline URMD & Residencial mediana densidad & 0 \\
\hline WATR & Cuerpos de agua & \\
\hline
\end{tabular}

Tabla 4. Profundidad total y de la primera capa de suelo para la cuenca del río Sordo. 
Tecnología y

Ciencias $₫$ Agua
2021, Instituto Mexicano de Tecnología del Agua

Open Access bajo la licencia CC BY-NC-SA 4.0

(https://creativecommons.org/licenses/by-nc-sa/4.0/)

\begin{tabular}{|c|c|c|c|}
\hline Clave & Suelo & $\begin{array}{c}\text { Profundidad, primera capa } \\
\text { WEAP (mm) }\end{array}$ & $\begin{array}{c}\text { Profundidad } \\
\text { total (mm) }\end{array}$ \\
\hline $\mathrm{AC}$ & Acrisol & 650 & 1100 \\
\hline $\mathrm{CM}$ & Cambisol & 600 & 1250 \\
\hline $\mathrm{EL}$ & Rendzina & 500 & 650 \\
\hline $\mathrm{FL}$ & Fluvisol & 500 & 1000 \\
\hline $\mathrm{HC}$ & Phaeozem & 450 & 650 \\
\hline $\mathrm{Is}$ & Litosol & 500 & 1000 \\
\hline $\mathrm{LC}$ & Luvisol & 550 & 800 \\
\hline $\mathrm{Re}$ & Regosol & 500 & 1000 \\
\hline $\mathrm{Vc}$ & Vertisol & 350 & 1000 \\
\hline
\end{tabular}

Con la profundidad radical ponderada y la profundidad de la capa profunda se calculó la capacidad de almacenamiento de agua en la zona radical $(S w)$ y la zona profunda $\left(D w_{s}\right)$ por tipo de suelo (Tabla 5$)$ a través de la Ecuación (5) y Ecuación (6). Finalmente, con la Ecuación (7) se obtuvieron los valores de la capacidad de almacenamiento en la zona profunda por subcuenca ( $\left.D w_{\text {sub }}\right)$; los valores oscilaron entre 83 y $294 \mathrm{~mm}$ :

$$
\begin{aligned}
& S W=\sum_{i=1}^{n} S O L_{-} Z_{i} S O L_{-} A W C_{i} \\
& D w_{S}=\sum_{i=1}^{n} S O L_{-} Z_{i} S O L_{-} A W C_{i}
\end{aligned}
$$


$D w_{\text {sub }}=\sum_{i=1}^{n} \frac{A_{i} D w_{s i}}{A T_{\text {sub }}}$

Donde $S w$ es la capacidad de almacenamiento de agua de la zona de raíces $(\mathrm{mm}) ; D w_{s}$ la capacidad de almacenamiento de agua en la zona profunda del tipo de suelo $(\mathrm{mm}) ; D w_{s u b}$, la capacidad de almacenamiento de agua de la subcuenca (mm); SOL_Z, la profundidad de la capa (mm); $S O L_{-} A W C_{i}$, la capacidad de agua disponible de la capa $\left(\mathrm{mm} \mathrm{mm}^{-1}\right) ; A_{i}$, el área del tipo de suelo (ha), y $A T_{\text {sub }}$ es el área total de la subcuenca (ha).

Tabla 5. Valores de la capacidad de almacenamiento de agua de la zona radical $(S w)$ y zona profunda $\left(D w_{s}\right)$ por tipo de suelo en la cuenca del río Sordo. 
Tecnología y

Ciencias $₫$ Agua
2021, Instituto Mexicano de Tecnología del Agua

Open Access bajo la licencia CC BY-NC-SA 4.0

(https://creativecommons.org/licenses/by-nc-sa/4.0/)

\begin{tabular}{|c|c|c|c|}
\hline Clave & Suelo & Sw (mm) & Dws (mm) \\
\hline AC & Acrisol & 340 & 340 \\
\hline CM & Cambisol & 274 & 274 \\
\hline EL & Rendzina & 291 & 291 \\
\hline FL & Fluvisol & 199 & 199 \\
\hline HC & Phaeozem & 205 & 205 \\
\hline Is & Litosol & 222 & 222 \\
\hline LC & Luvisol & 296 & 296 \\
\hline Re & Regosol & 212 & 212 \\
\hline Vc & Vertisol & 178 & 178 \\
\hline
\end{tabular}

El índice de área foliar (LAI, por sus siglas en inglés), localizado en el tercer término de la Ecuación (1) en WEAP, representa el efecto del dosel en el escurrimiento superficial. Este valor se tomó de la calibración de SWAT, donde se le identifica como índice de área foliar máximo $\left(\mathrm{m}^{2}\right.$ $\mathrm{m}^{-2}$ ), BLAI (Tabla 6).

Tabla 6. Índices de área foliar por tipo de cobertura ingresados a WEAP para la cuenca del río Sordo.

\begin{tabular}{|l|l|c|}
\hline Clave & Descripción & IAF o RRF $\left(\mathbf{m}^{\mathbf{2}} \mathbf{~ m}^{\mathbf{2}} \mathbf{)}\right.$ \\
\hline BENC & Bosque de encino & 5.7 \\
\hline
\end{tabular}


Tecnología y

Ciencias $₫$ Agua
2021, Instituto Mexicano de Tecnología del Agua

Open Access bajo la licencia CC BY-NC-SA 4.0

(https://creativecommons.org/licenses/by-nc-sa/4.0/)

\begin{tabular}{|l|l|c|}
\hline Clave & Descripción & IAF o RRF $\left(\mathbf{m}^{\mathbf{2}} \mathbf{~ m}^{-2}\right)$ \\
\hline ENPI & Bosque de encino-pino & 5.7 \\
\hline FRSD & Selva baja caducifolia & 2.1 \\
\hline FRSE & Bosque mesófilo de montaña & 5.6 \\
\hline MATO & Chaparral & 2.1 \\
\hline PASI & Pastizal & 1.7 \\
\hline PIEN & Bosque de pino-encino & 5.5 \\
\hline PINO & Bosque de pino & 5.5 \\
\hline RIEG & Agricultura de riego & 3.6 \\
\hline RNGB & Bosque de táscate & 5.6 \\
\hline TEMP & Agricultura de temporal & 3.6 \\
\hline URMD & Residencial mediana densidad & 8 \\
\hline WATR & Cuerpos de agua & 0.1 \\
\hline
\end{tabular}

La conductividad hidráulica a saturación en la zona radical $(K s)$ y en la zona profunda $(K d)$ se presenta cuando el almacenamiento relativo en Z1 y Z2, respectivamente, es igual a 1.0 (saturación). El valor de Ks se divide de acuerdo con la dirección preferencial del flujo, en forma subsuperficial y percolación hacia la capa profunda. Mientras que $K d$ controla el movimiento del flujo base, de modo que éste se incrementa a medida que la $K d$ aumenta. 
Con la Ecuación (8) y Ecuación (9) se obtuvieron, por tipo de suelo, los valores de $K s$ y $K d_{s}$ (Tabla 7$)$, respectivamente. La información de conductividad hidráulica proviene de la corrida previa con SWAT (Sánchez-Galindo et al., 2017). Sin embargo, como el parámetro de conductividad en la zona profunda se ingresó a nivel subcuenca $\left(K d_{\text {sub }}\right)$, éste se calculó con la Ecuación (10), obteniéndose un rango entre 10 y 1 $241 \mathrm{~mm} \mathrm{mes}^{-1}$.

$K s=\sum_{i=1}^{n} \frac{S O L_{-} Z_{i} K 24_{i}}{P r_{1}}$

$K d_{s}=\sum_{i=1}^{n} \frac{S O L_{-} Z_{i} K 24_{i}}{P r_{2}}$

$$
K d_{\text {sub }}=\sum_{i=1}^{n} \frac{A_{i} K d_{S_{i}}}{A T_{\text {sub }}}
$$

Donde $K s$ es la conductividad hidráulica de la zona radical ( $\mathrm{mm}$ mes$\left.{ }^{1}\right) ; K d_{s}$, la conductividad hidráulica de la zona profunda del tipo de suelo ( $\left.\mathrm{mm} \mathrm{mes}^{-1}\right) ; K d_{\text {sub }}$, la conductividad hidráulica de la zona profunda de la subcuenca $\left(\mathrm{mm} \mathrm{mes}{ }^{-1}\right) ; S O L \_Z$, la profundidad de la capa $(\mathrm{mm}) ; K 24 i$, el parámetro de SWAT SOL_K de la capa multiplicado por 24; $P r_{1}$, la profundidad radicular por tipo de suelo ponderada $(\mathrm{mm}) ; \quad P r_{2}$, la profundidad de la capa profunda por tipo de suelo (mm); $A_{i}$, el área del tipo de suelo (ha), y $A T_{\text {sub }}$ es el área total de la subcuenca (ha). 
Tecnología y

Ciencias $\cong$ Agua
2021, Instituto Mexicano de Tecnología del Agua

Open Access bajo la licencia CC BY-NC-SA 4.0

(https://creativecommons.org/licenses/by-nc-sa/4.0/)

Tabla 7. Valores de conductividad hidráulica en la zona radical ( $K s)$ y profunda $\left(K d_{s}\right)$ por tipo de suelo, para la cuenca del río Sordo.

\begin{tabular}{|c|c|c|c|}
\hline Clave & Suelo & Ks $\left(\mathrm{mm} \mathrm{mes}{ }^{-1}\right)$ & $\left.K d_{s}(\mathrm{~mm} \mathrm{mes})^{-1}\right)$ \\
\hline$A C$ & Acrisol & 32 & 28 \\
\hline CM & Cambisol & 569 & 318 \\
\hline$\overline{E L}$ & Rendzina & 796 & 10 \\
\hline $\mathrm{FL}$ & Fluvisol & 1064 & 1331 \\
\hline $\mathrm{HC}$ & Phaeozem & 278 & 94 \\
\hline Is & Litosol & 335 & 10 \\
\hline LC & Luvisol & 483 & 212 \\
\hline $\operatorname{Re}$ & Regosol & 540 & 10 \\
\hline Vc & Vertisol & 121 & 123 \\
\hline
\end{tabular}

La humedad de la zona radicular (Z1) y de la zona profunda (Z2) al inicio de la simulación es el almacenamiento relativo de la primera y segunda capa, respectivamente, expresado como porcentaje de la acumulación efectiva total. Se ingresó el 30 \% para ambas humedades. 


\section{Evaluación de la eficiencia}

La evaluación de la eficiencia del modelo WEAP para simular escurrimientos anuales y mensuales se llevó a cabo durante el periodo 1975-1985, siendo 1975 el año base.

La evaluación del comportamiento y el desempeño de los modelos se hizo comparando el escurrimiento simulado y el escurrimiento medido en la salida del área de captación (Krause, Boyle, \& Bäse, 2005). Los índices utilizados en el presente trabajo se describen a continuación.

El coeficiente de determinación $\left(r^{2}\right)$ describe la variación de los datos observados con respecto a los simulados por el modelo. El rango de $r^{2}$ varía de 0 a 1 ; los valores más altos indican un menor error de variación y valores mayores a 0.5 se consideran aceptables. Este estadístico es demasiado sensible a los valores extremos altos e insensible a las diferencias aditivas y proporcionales entre las predicciones del modelo y los datos medidos (Moriasi et al., 2007).

El Índice de eficiencia de Nash y Sutcliffe (NSE), es el estadístico normalizado que determina la magnitud relativa de la varianza residual (ruido), en comparación con la variación de los datos medidos (información). Indica qué tan bien la gráfica de datos observados versus 
simulados se ajusta a la línea 1:1. Toma valores comprendidos entre - $\infty$ y 1 ; si el resultado es 1 , el ajuste es perfecto; si es 0 , el error es del mismo orden de magnitud que la varianza de los datos observados, por lo que la media de los datos observados tendrá una capacidad de predicción similar al modelo. Valores inferiores a cero implican que la media tiene una capacidad de predicción más alta que el modelo, lo cual implica que los valores simulados son malos (Moriasi et al., 2007).

El sesgo porcentual (PBIAS) calcula la tendencia del modelo a subestimar (valores positivos) o sobreestimar (valores negativos) la variable de interés. Los valores de baja magnitud indican una simulación precisa del modelo, siendo 0 el número óptimo (Moriasi et al., 2007).

\section{Resultados y discusión}

\section{Caudales mensuales}


Como parte de los resultados se presentan los caudales mensuales y anuales simulados con SWAT (Sánchez-Galindo et al., 2017) y los generados con WEAP a través de parámetros biofísicos calibrados en SWAT para la cuenca del río Sordo.

En la Figura 4 se muestran los caudales mensuales medidos versus los simulados por SWAT y WEAP para el periodo 1976 a 1985; también se incluyen los valores de NSE y PBIAS. Se observa que WEAP calculó bien los caudales base mientras que SWAT, aunque replicó de buena manera los caudales pico, presentó problemas con la curva de recesión al aproximarse al caudal base. Este comportamiento de WEAP difiere de los resultados obtenidos por Ingol-Blanco y McKinney (2013), quienes encontraron que WEAP reproduce mejor los escurrimientos pico que los caudales base en la cuenca del río Conchos. Asimismo, se nota que el valor de NSE de SWAT (0.82) fue mayor que el obtenido en WEAP (0.73). Esto implica, de acuerdo con Moriasi et al. (2007), que los valores de NSE son "muy bueno" para SWAT y "bueno" para WEAP. 
Tecnología y

Ciencias $\cong$ Agua
2021, Instituto Mexicano de Tecnología del Agua

Open Access bajo la licencia CC BY-NC-SA 4.0

(https://creativecommons.org/licenses/by-nc-sa/4.0/)

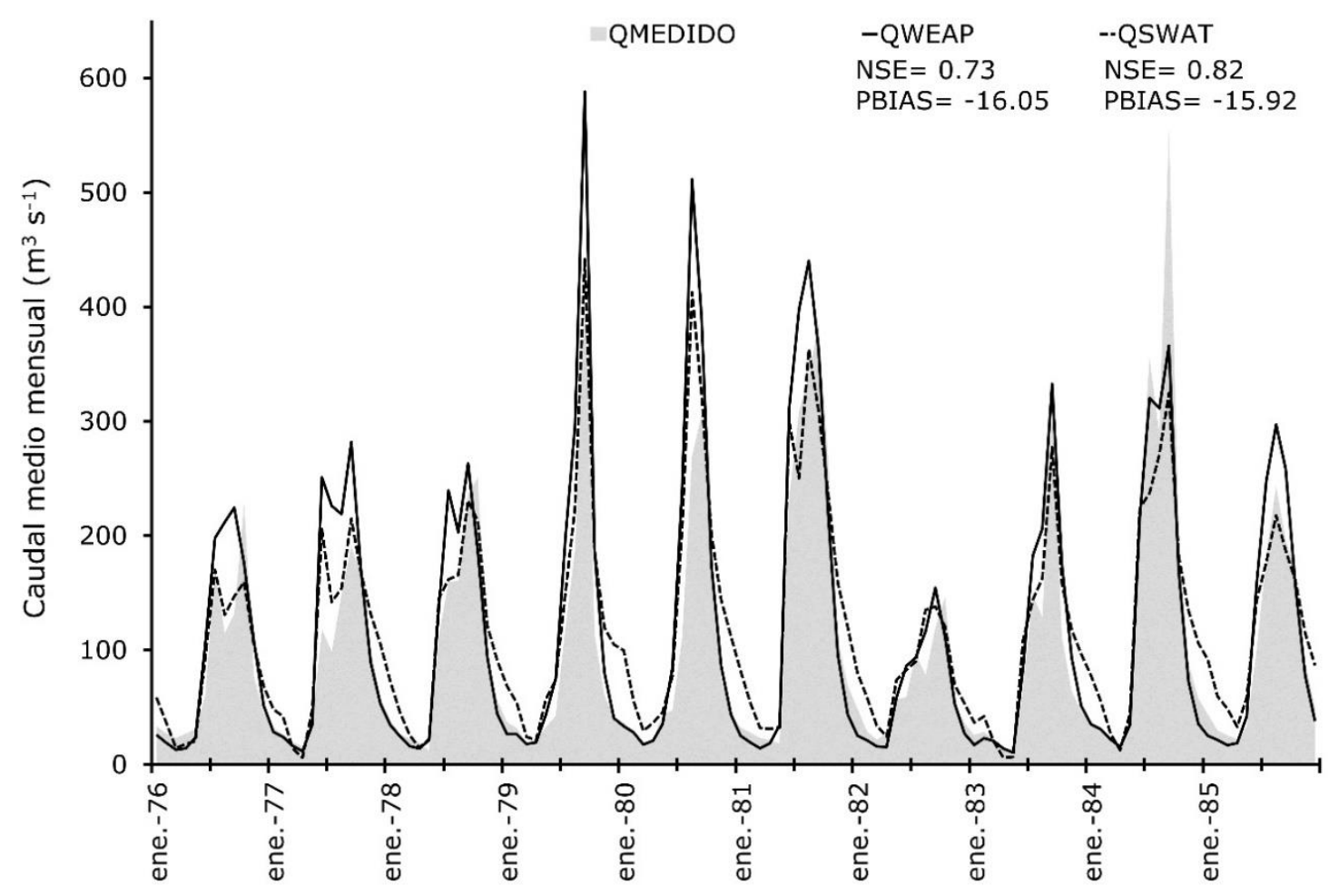

Figura 4. Caudales medios mensuales observados y simulados por SWAT y WEAP de la cuenca del río Sordo.

El índice NSE $=0.73$ de WEAP se encuentra dentro del rango de resultados que han tenido otros autores, como Varela-Ortega et al. (2016) en la cuenca del río Guadiana, España, con NSE > 0.7; Olsson et al. (2017), en la cuenca Chancay-Huaral Perú, con NSE $\geq 0.8$; y Höllermann, Giertz y Diekkrüger (2010), en la cuenca Ouémé-Bonou, Benín, con NSE $\geq 0.78$. 
Por otro lado, los valores de PBIAS -16.05 y -15.92 de WEAP y $S W A T$, respectivamente, indican que ambas herramientas sobreestiman de manera similarel caudal mensual observado.

En la Figura 5 se observa que el $r^{2}$ del escurrimiento medio mensual de SWAT fue ligeramente más alto que el de WEAP (0.85 vs. 0.84), es decir, revela menor error de variación. Sin embargo, aunque la $r^{2}$ se ha utilizado ampliamente para la evaluación de modelos, sólo cuantifica la dispersión de los resultados. Por ejemplo, un modelo que sistemáticamente sobreestima o subestima todo el tiempo mostrará valores cercanos a 1.0, incluso si todas las predicciones fueran erróneas (Krause et al., 2005; Moriasi et al., 2007). Sin embargo, al considerar el valor de $r^{2}$, respecto a la ordenada al origen, se observa que WEAP presenta un valor más cercano a cero (6.6) que SWAT (34.9); esto es, un caudal observado de 0 daría como resultado 6.6 en WEAP y 34.9 en SWAT. Al mismo tiempo, la pendiente de la recta refleja una sobrepredicción de $9.55 \%$ para WEAP y una subpredicción de $15.32 \%$ para SWAT. 
Tecnología y

Ciencias $\stackrel{\varpi}{\triangleleft}$ gua
2021, Instituto Mexicano de Tecnología del Agua

Open Access bajo la licencia CC BY-NC-SA 4.0

(https://creativecommons.org/licenses/by-nc-sa/4.0/)

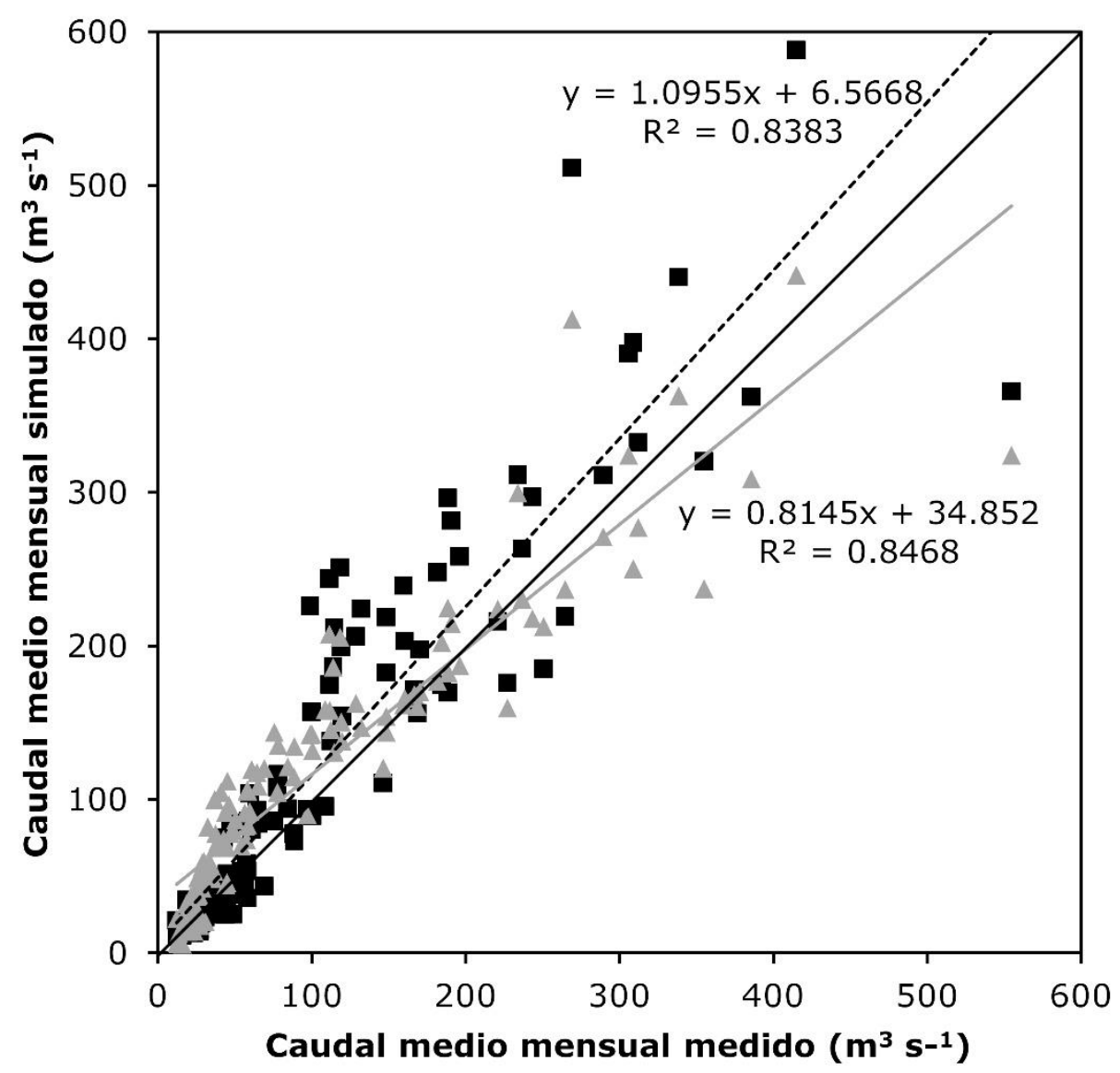

-QWEAP $\triangle$ QSWAT --Lineal (QWEAP) -Lineal (QSWAT)

Figura 5. Relación entre caudales medios mensuales observados y simulados con SWAT y WEAP en la cuenca del río Sordo.

En 2016, Adgolign et al. evaluaron el cambio de la disponibilidad de agua en la subcuenca Didessa, Etiopía. Para ello, se apoyaron de las mismas herramientas que en el presente trabajo, SWAT y WEAP. Con SWAT se completaron los huecos en los datos de flujo medidos; mientras 
que WEAP se utilizó para modelar la asignación de los recursos hídricos superficiales de la cuenca.

Hussen et al. (2018) calibraron y validaron la capacidad de SWAT para simular el escurrimiento de la subcuenca Abaya-Chamo, Etiopía, obteniendo un $r^{2}=0.77$ y un NSE $=0.76$ durante la calibración, y un $r^{2}$ $=0.80$ y un NSE $=0.78$ en la validación. Posteriormente se implementó el modelo WEAP para asignar los recursos hídricos de la subcuenca bajo escenarios de cambio climático.

En la cuenca del sur de Phuthiatsana, Lesoto, se evaluó la cantidad de agua superficial. Para ello se estimaron flujos en cuencas no aforadas con SWAT y se asignaron los recursos en la cuenca utilizando WEAP. SWAT se calibró de 1979 a 2001, NSE $=0.59$ y $r^{2}=0.59$, validó de 2002 a 2013, NSE $=0.52$ y $r^{2}=0.66$ (Maliehe \& Mulungu, 2017).

\section{Caudales medios anuales}

Los caudales medios anuales simulados en WEAP alcanzaron un NSE = 0.3 al compararse con los valores observados en la estación hidrométrica, 
resultando un ajuste "insatisfactorio" (Moriasi et al., 2007), sobre todo por el efecto de los años 1977, 1979 y 1980 (Figura 6). Por su parte, SWAT alcanzó un mejor ajuste con un NSE $=0.73$ "bueno" y un PBIAS $=$ -4.6, es decir sólo sobreestimó en un $4.6 \%$. Algo semejante ocurrió con los valores de $r^{2}$ : WEAP obtuvo 0.63 mientras que SWAT logró 0.76 (Figura 7).

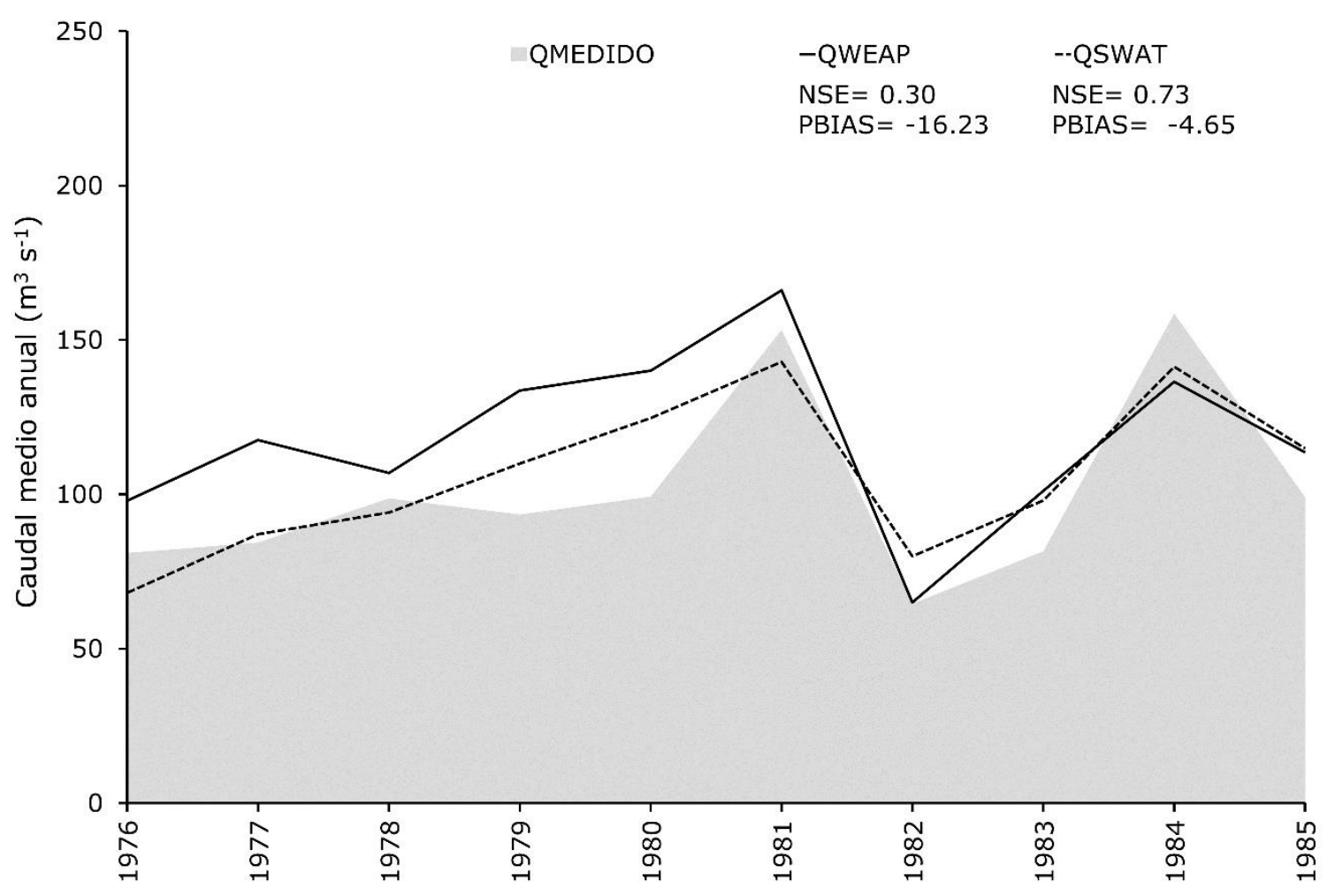

Figura 6. Caudales medios anuales observados y simulados por SWAT y WEAP de la cuenca del río Sordo. 
Teçnología y

Ciencias $₫$ Agua
2021, Instituto Mexicano de Tecnología del Agua

Open Access bajo la licencia CC BY-NC-SA 4.0

(https://creativecommons.org/licenses/by-nc-sa/4.0/)

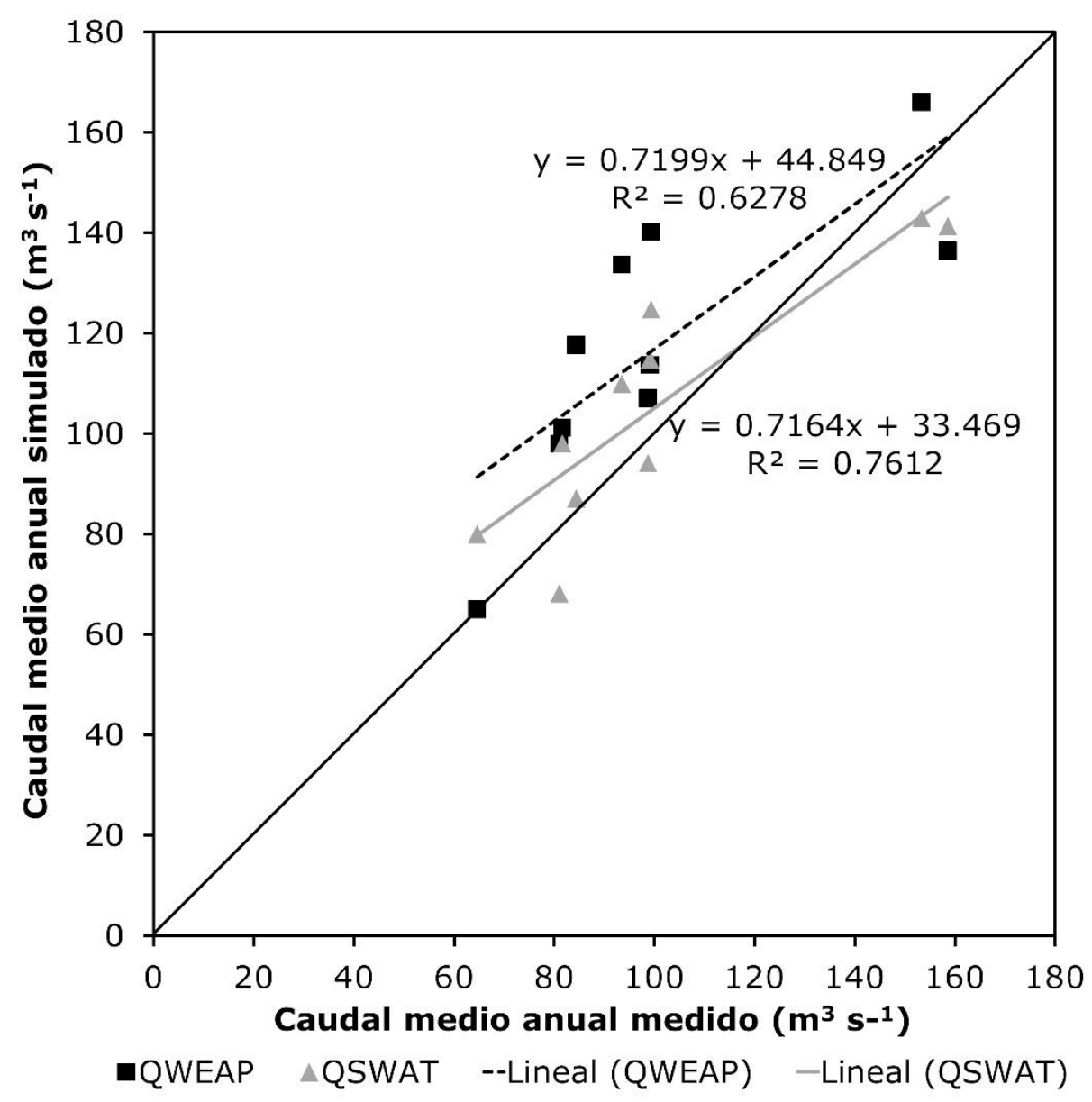

Figura 7. Relación entre caudales medios anuales observados y simulados por SWAT y WEAP de la cuenca del río Sordo.

En la Tabla 8 es posible apreciar que SWAT y WEAP son confiables para la simulación de escurrimientos mensuales y anuales, según los índices de $r^{2}$, NSE y PBIAS, aseveración también estipulada por Faiz et al. (2018), quienes obtuvieron para WEAP valores de NSE entre 0.83 y 0.88 , 
y $r^{2}$ entre 0.86 y 0.92 ; y para SWAT, valores de NSE entre 0.80 y 0.81 y $r^{2}$ entre 0.81 y 0.82 . Asimismo, en el trabajo de Sánchez-Galindo et al. (2017), SWAT simuló satisfactoriamente la producción de biomasa y sedimentos. Es importante resaltar que en este trabajo se aprovechó la información previamente recabada y utilizada para alimentara SWAT, sin embargo, es totalmente factible alimentar WEAP sin modelar previamente en SWAT y hasta podría considerarse más sencillo debido a la naturaleza más robusta de WEAP. Por ende, la elección de uno u otro modelo dependerá de los datos disponibles, los objetivos del estudio, de la herramienta que conozca el modelador y de las salidas de interés de los tomadores de decisiones.

Tabla 8. Evaluación de la eficiencia para simular escurrimientos mensuales y anuales de WEAP y SWAT en la cuenca del río Sordo.

\begin{tabular}{|l|l|c|c|l|c|l|}
\hline Periodo & Modelo & $\boldsymbol{r}^{\mathbf{2}}$ & NSE & Ajuste NSE & PBIAS (\%) & Ajuste PBIAS \\
\hline \multirow{2}{*}{ Mensual } & SWAT & 0.85 & 0.82 & Muy bueno & -15.92 & Satisfactorio \\
\cline { 2 - 7 } & WEAP & 0.84 & 0.73 & Bueno & -16.05 & Satisfactorio \\
\hline \multirow{2}{*}{ Anual } & SWAT & 0.76 & 0.73 & Bueno & -4.65 & Muy bueno \\
\cline { 2 - 7 } & WEAP & 0.63 & 0.3 & Insatisfactorio & -16.23 & Satisfactorio \\
\hline
\end{tabular}


Tecnología y

Ciencias Agua
2021, Instituto Mexicano de Tecnología del Agua

Open Access bajo la licencia CC BY-NC-SA 4.0

(https://creativecommons.org/licenses/by-nc-sa/4.0/)

\section{Conclusiones}

La modelación hidrológica es una herramienta útil para el conocer el comportamiento y la distribución de los recursos hídricos en una cuenca. El conocimiento que se obtiene de este proceso es crucial en la implementación de políticas de gestión sustentable para el uso y aprovechamiento del agua.

Los resultados obtenidos en el presente estudio manifiestan que los modelos SWAT y WEAP son capaces de simular satisfactoriamente los escurrimientos mensuales y anuales de la cuenca del río Sordo, según los índices de eficiencia $r^{2}$, NSE y PBIAS. Sin embargo, en la escala de tiempo anual, WEAP presenta un NSE $=0.3$, por lo cual SWAT fue superior.

La cantidad de datos para el funcionamiento de estas dos herramientas es desigual. Por un lado, SWAT, modelo de base física, requiere una enorme cantidad de información; mientras que WEAP, modelo conceptual-base física, demanda menor cantidad de datos. Sin embargo, esta característica a favor de WEAP presenta el inconveniente de que los valores de esos pocos parámetros que lo definen no están plenamente acotados en la literatura, como sucede con SWAT. 
De este trabajo podemos deducir que es posible obtener resultados satisfactorios en WEAP utilizando datos de la herramienta SWAT, como se ha realizado en otras investigaciones. Sin embargo, sólo se recomienda recurrir a SWAT para alimentar a WEAP cuando se cuente con un trabajo de éste en el sitio de interés.

Es importante enfatizar que antes de elegir el mejor modelo se deben tener claros los objetivos del estudio, al igual que la disponibilidad, capacidades y necesidades de la herramienta computacional a utilizar.

\section{Referencias}

Adgolign, T. B., Srinivasa-Rao, G. V. R., \& Abbulu, Y. (2016). WEAP modeling of surface water resources allocation in Didessa Sub-Basin, West Ethiopia. Sustainable Water Resources Management, 2, 55-70. Recuperado de https://doi.org/10.1007/s40899-015-0041-4

Allen, R. G., Pereira, L. S., Raes, D., \& Smith, M. (2006). Estudio FAO riego y drenaje 56. Evapotranspiración del cultivo (guías para la determinación de riego de los requerimientos de agua de los cultivos). Roma, Italia: Food and Agriculture Organization of the United Nations. Recuperado de http://www.fao.org/3/a-x0490s.pdf

Angarita, H., Wickel, A. J., Sieber, J., Chavarro, J., Maldonado-Ocampo, J. A., Herrera-R., G. A., Delgado, J., \& Purkey, D. (2018). Basin-scale impacts of hydropower development on the Mompós Depression wetlands, Colombia. Hydrology and Earth System Sciences, 22(5), 
2839-2865. Recuperado de https://doi.org/10.5194/hess-22-28392018

Conagua-IMTA, Comisión Nacional del Agua-Instituto Mexicano de Tecnología del Agua. (2019). Banco Nacional de Datos de Aguas Superficiales (BANDAS). Recuperado de https://www.imta.gob.mx/bandas

Cuceloglu, G., Abbaspour, K. C., \& Ozturk, I. (2017). Assessing the waterresources potential of Istanbul by using a soil and water assessment tool (SWAT) hydrological model. Water (Switzerland), 9(10), 814832. Recuperado de https://doi.org/10.3390/w9100814

Faiz, M. A., Liu, D., Fu, Q., Li, M., Baig, F., Ahmad-Tahir, A., Imran-Khan, M., Li, T., \& Cui, S. (2018). Performance evaluation of hydrological models using ensemble of General Circulation Models in the northeastern China. Journal of Hydrology, 565, 599-613. Recuperado de https://doi.org/10.1016/j.jhydrol.2018.08.057

Hernández-Vargas, M. Á. (2017). Implementación de la herramienta WEAP al sistema Cutzamala. Ciudad de México, México: Universidad Nacional Autónoma de México.

Höllermann, B., Giertz, S., \& Diekkrüger, B. (2010). Benin 2025Balancing future water availability and demand using the WEAP "Water Evaluation and Planning" System. Water Resources Management, 24(13), 3591-3613. Recuperado de https://doi.org/10.1007/s11269-010-9622-z

Hussen, B., Mekonnen, A., \& Pingale, S. M. (2018). Integrated water 
resources management under climate change scenarios in the subbasin of Abaya-Chamo, Ethiopia. Modeling Earth Systems and Environment, 4(1), 221-240. Recuperado de https://doi.org/10.1007/s40808-018-0438-9

Ingol-Blanco, E., \& McKinney, D. C. (2013). Development of a hydrological model for the rio Conchos Basin. American Society of Civil Engineers, 18, 340-351. Recuperado de https://doi.org/10.1061/(ASCE)HE.1943-5584.0000607.

Krause, P., Boyle, D. P., \& Bäse, F. (2005). Comparison of different efficiency criteria for hydrological model assessment. Advances in Geosciences, 5, 89-97. Recuperado de https://doi.org/10.5194/adgeo-5-89-2005

Maliehe, M., \& Mulungu, D. M. M. (2017). Assessment of water availability for competing uses using SWAT and WEAP in South Phuthiatsana catchment, Lesotho. Physics and Chemistry of the Earth, 100, 305316. Recuperado de https://doi.org/10.1016/j.pce.2017.02.014

Moriasi, D. N., Arnold, J. G., Van Liew, M. W., Bingner, R. L., Harmel, R. D., \& Veith, T. L. (2007). Model evaluation guidelines for systematic quantification of accuracy in watershed simulations. American Society of Agricultural and Biological Engineers, 50(3), 885-900.

NCEP, The National Centers for Environmental Prediction. (2019). Global Weather Data for SWAT. Recuperado de https://globalweather.tamu.edu/

Olsson, T., Kämäräinen, M., Santos, D., Seitola, T., Tuomenvirta, H., 
Haavisto, R., \& Lavado-Casimiro, W. (2017). Downscaling climate projections for the Peruvian coastal Chancay-Huaral Basin to support river discharge modeling with WEAP. Journal of Hydrology: Regional Studies, 13 , 26-42. Recuperado

de https://doi.org/10.1016/j.ejrh.2017.05.011

Salgado, J. H., \& Güitrón, A. (2012). Aplicabilidad de los modelos hidrológicos distribuidos. En: XXII Congreso Nacional de Hidráulica (p. 5), Acapulco, Guerrero, México.

Sánchez-Galindo, M., Fernández-Reynoso, D. S., Martínez-Ménez, M. R., Rubio-Granados, E., \& Ríos-Berber, J. D. (2017). Modelo hidrológico de la cuenca del río Sordo, Oaxaca, México, con SWAT. Tecnología y ciencias del agua, 8(5), 141-156. Recuperado de https://doi.org/10.24850/j-tyca-2017-05-10

Sieber, J., \& Purkey, D. (2015). User Guide for WEAP 2015. Recuperado de https://www.weap21.org/downloads/WEAP_User_Guide.pdf

Singh, V. P., \& Woolhiser, D. A. (2002). Mathematical modeling of watershed hydrology. Journal of Hydrologic Engineering, 7(4), 270292. Recuperado de https://doi.org/10.1061/(asce)10840699(2002)7:4(270)

Varela-Ortega, C., Blanco-Gutiérrez, I., Esteve, P., Bharwani, S., Fronzek, S., \& Downing, T. E. (2016). How can irrigated agriculture adapt to climate change? Insights from the Guadiana basin in Spain. Regional Environmental Change, 16(1), 59-70. Recuperado de https://doi.org/10.1007/s10113-014-0720-y 
2021, Instituto Mexicano de Tecnología del Agua

Ciencias $₫$

Open Access bajo la licencia CC BY-NC-SA 4.0

(https://creativecommons.org/licenses/by-nc-sa/4.0/)

Yates, D., Sieber, J., Purkey, D., \& Huber-Lee, A. (2005). WEAP21-A Demand-, Priority-, and Preference-Driven Water Planning Model. Part 1 : Model Characteristics. Water International, 30(4), 487-500. 